\title{
Comparison of Global Optimization Methods for Insertion Maneuver into Earth-Moon L2 Quasi-Halo Orbit Considering Collision Avoidance
}

\author{
Sang-Cherl Lee*, Hae-Dong Kim*, Do-Chul Yang*, Dong-Hyun Cho* and Jeong-Heum Im* \\ Korea Aerospace Research Institute, Daejeon, Republic of Korea
}

\section{Tae-Soo No**}

Department of Aerospace Engineering, Chonbuk National University, Jeonju, Republic of Korea

\author{
Seungkeun Kim*** and Jinyoung Suk**** \\ Department of Aerospace Engineering, Chungnam National University, Daejeon, Republic of Korea
}

\begin{abstract}
A spacecraft placed in an Earth-Moon L2 quasi-halo orbit can maintain constant communication between the Earth and the far side of the Moon. This quasi-halo orbit could be used to establish a lunar space station and serve as a gateway to explore the solar system. For a mission in an Earth-Moon L2 quasi-halo orbit, a spacecraft would have to be transferred from the Earth to the vicinity of the Earth-Moon L2 point, then inserted into the Earth-Moon L2 quasi-halo orbit. Unlike the near Earth case, this orbit is essentially very unstable due to mutually perturbing gravitational attractions by the Earth, the Moon and the Sun. In this paper, an insertion maneuver of a spacecraft into an Earth-Moon L2 quasi-halo orbit was investigated using the global optimization algorithm, including simulated annealing, genetic algorithm and pattern search method with collision avoidance taken into consideration. The result shows that the spacecraft can maintain its own position in the Earth-Moon L2 quasi-halo orbit and avoid collisions with threatening objects.
\end{abstract}

Key words: Circular Restricted Three Body Problem, Quasi-Halo, Collision Avoidance, Space Objects, Simulated Annealing, Genetic Algorithm, Pattern Search, Maneuver Planning, Orbit Insertion Maneuver

\section{Introduction}

In 1760, Leonard Euler formulated the restricted three body problem in a rotating frame handmade prediction on the premise that three collinear equilibrium points (L1, L2, L3) exist. In his 1772 essay "essaisur le probleme des Trois Corps", Lagrange confirmed Euler's prediction and solved for two additional equilibrium points (L4, L5) which form an equilateral triangle with the primaries. Henri Poincare later demonstrated in his "Methodes Nouvelles de la Mechanique Celeste" that there could be no such analytic quantities in positions, velocities and mass ratio. He concluded that the three body problem could not be solved using algebraic formulas and integrals[1]. The Sun-Earth and Earth-Moon Lagrange points as well as their Halo orbits are shown in Fig.1.

The triangular equilibrium points, L4 and L5, could be used as parking regions because no station keeping is necessary on those points. On the other hand, the collinear points L1, L2 and L3 can be selected for space observation and planetary exploration missions. They can provide good observation orbits toward the Sun in the Sun-Earth system during Sun observation missions. A spacecraft for the space observation mission orbiting around the Sun-Earth L2 point can provide constant observation geometry with half of the entire celestial
This is an Open Access article distributed under the terms of the Creative Commons Attribution Non-Commercial License (http://creativecommons.org/licenses/by$\mathrm{nc} / 3.0 /$ which permits unrestricted non-commercial use, distribution, and reproduction in any medium, provided the original work is properly cited.

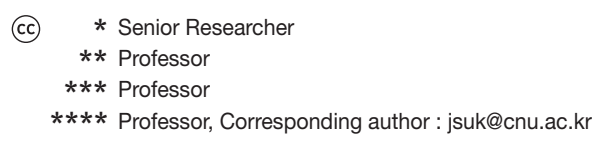


sphere available at any given time. Additionally, an orbit around the Sun-Earth L2 point is useful for non-cryogenic missions that require great thermal stability, suitable for highly precise visible light telescopes. Periodic orbits around the Earth-Moon L2 point can be used to establish a permanent communication link between the Earth and the far side of the Moon as suggested by A.C. Clark in 1950 and R. Farquhar in 1966. An orbit around a Lagrange point provides the ability for Earth transfer, return trajectory and interplanetary transport. Recent studies have also demonstrated that even formation flying is possible using the Lagrange points[2].

Table 1 lists past, present and future spacecraft missions on the Lagrange points[3]. The ARTEMIS mission was the first to navigate and perform station keeping operations on the Earth-Moon L1 and L2 Lagrange points. ARTEMIS P1 and P2 spacecrafts were used for simultaneous measurements of particles and electromagnetic fields from the two locations.
They provided the first ever three-dimensional information on how energetic particle acceleration takes place near the Moon's orbit in the distant magnetosphere and in the solar wind[4].

Roughly 50 man-made objects had been placed in the lunar orbit since the dawn of space exploration up to mid2005. At least 34 of them have crashed onto the Moon or been maneuvered out of the lunar orbit. Several of the remaining spacecrafts and propulsion units are also believed to have reached the lunar surface[5]. Although the number of manmade debris or asteroids in the lunar orbit is still small, a collision avoidance maneuver system would be useful to reduce the probability of colliding with uncontrolled threatening objects from the outer space.

The scope of this paper focuses on an insertion maneuver to the quasi-halo orbit using global optimization methods including simulated annealing, genetic algorithm and pattern search. Prior works related to this research are

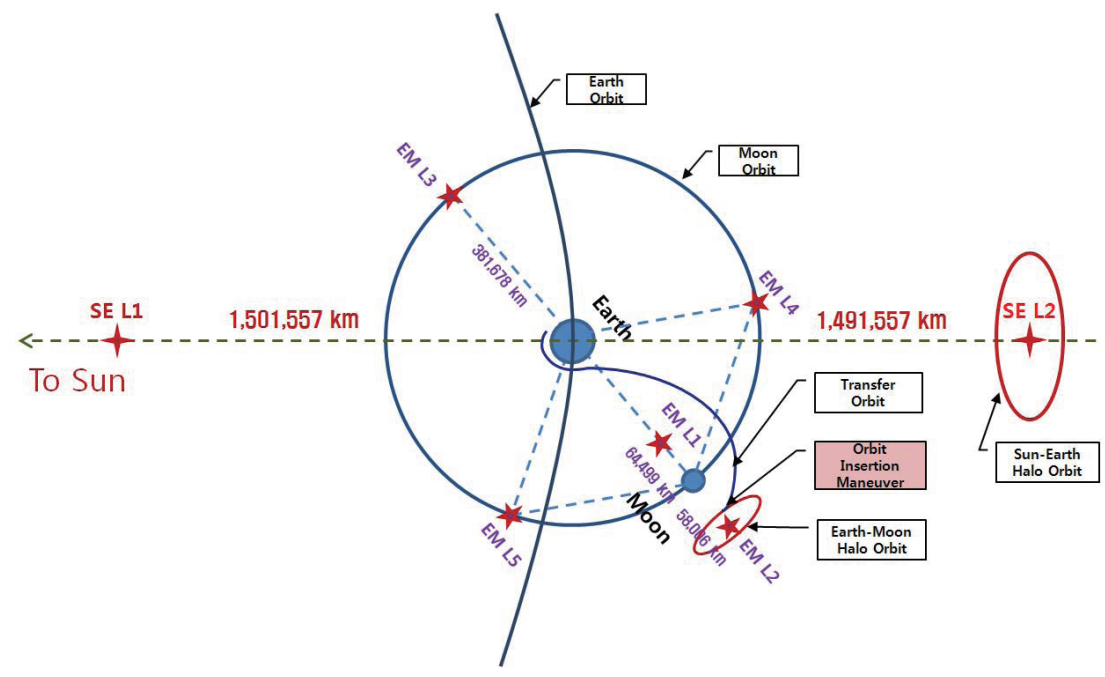

Fig. 1. Lagrange Point Orbits and Halo orbits

Table 1. Spacecraft Missions on the Lagrange Points

\begin{tabular}{|c|c|c|c|}
\hline Point & Status & Earth-Sun & Earth-Moon \\
\hline \multirow{3}{*}{ L1 } & $\begin{array}{l}\text { Mission } \\
\text { Completed }\end{array}$ & ISEE-3, Genesis & Not reported \\
\hline & On Operation & WIND, SOHO, ACE & Not reported \\
\hline & Planned & $\begin{array}{c}\text { LISA Pathfinder, KUAFU, Deep Space Climate } \\
\text { Observatory, Solar-C }\end{array}$ & Not reported \\
\hline \multirow{3}{*}{ L2 } & $\begin{array}{c}\text { Mission } \\
\text { Completed }\end{array}$ & WIND, Chang'e2 & ARTEMIS P1/P2 \\
\hline & On Operation & WMAP, Planck Surveyor, Herschel Space Observatory & Not reported \\
\hline & Planned & $\begin{array}{c}\text { JWST, Gaia, Terrestrial Planet Finder, Darwin, Wide Field } \\
\text { Infrared Survey Telescope, Euclid }\end{array}$ & Exploration Gateway Platform \\
\hline L4 & On Operation & STEREO A & Not reported \\
\hline L5 & On Operation & STEREO B & Not reported \\
\hline
\end{tabular}


described as follows. Santos et al. studied the transfer trajectories between two coplanar orbits using several impulses, using genetic algorithms to find solutions that minimize fuel consumption[6]. Paluszek et al. used a downhill simplex, genetic algorithm and simulated annealing to investigate the problem of finding a minimum time trajectory from the Earth to the Mars orbit using a fixed thrust electric propulsion system[7]. Wang et al. implemented the pattern search method to reduce deviations between calculations performed by the onboard calculation and the ground elaboration orbit models with the goal of improving the accuracy of on-board attitude determination for satellites[8]. Vries developed an efficient method of satellite maneuver optimization based on a Monte Carlo approach in combination with simulated annealing[9]. Lastly, Kim et al. studied a genetic algorithm optimization of the lunar transfer trajectory using a weak stability boundary[10].

This paper investigates an insertion maneuver of a spacecraft into the Earth-Moon L2 quasi-halo orbit by using simulated annealing, genetic algorithm and pattern search method to decrease the probability of collision with threatening uncontrolled objects. The fitness function is used to minimize the distance between the reference L2 quasi-halo orbit and a maneuvering spacecraft. The STK/ Astrogator, STK/AdvCat and Matlab global optimization toolbox are utilized as simulation tools[12, 22].

Unlike the aforementioned works, this study aims to compare global optimization methods for an insertion maneuver into the Earth-Moon L2 quasi-halo orbit with collision avoidance taken into consideration. The most valuable benefit of this approach is that it can get a periodic orbit trajectory near the unstable Lagrange points where the gravitational attractions of the Earth and Moon equalize.

The rest of this paper is organized as follows. Section 2 contains a brief introduction of the optimization algorithm used in this paper. The circular restricted three body problem is described in Section 3. Section 4 presents the maneuver plan for an insertion into the Earth-Moon L2 quasi-halo orbit using global optimization. Sections 5 and 6 describe simulation results and the conclusion.

\section{Global Optimization Algorithm}

The non-linear solver based on the gradient method quickly converges to a local minimum near the initial guess, but may not find a global solution. In contrast, the global search method is able to search more effectively within an entire solution space and find a global minimum[12]. This paper compares three global optimization algorithms. The simulated annealing method uses stochastic iteration or meta-heuristics which is derivative-free and can handle problems without constraints or boundary conditions. This method can find a global solution given a slowenough cooling schedule. The genetic algorithm is based on evolutionary computation and also uses stochastic iteration without gradients. The pattern search method uses deterministic iteration, which is known to be more robust and efficient in computation time.

The simulated annealing method is a generic probabilistic meta-heuristic method proposed by Kirkpatrick, Gelett and Vecchiinin in 1983 to find a global minimum of a cost function. The method originated from thermodynamics and models the cooling process of a metal, with the initial state fed into a neighbor-generating function to create a random neighboring state. Any downhill step is accepted for minimizing functions, and the process is repeated from a new point afterward. If an uphill step is accepted, the simulated annealing can escape from a local minimum. This uphill decision is made using the Metropolis criteria. As optimization progresses, the length of the steps decreases and the solution gets closer to a global optimum $[9,12]$.

The Genetic algorithm introduced in 1975 by John Holland is a meta-heuristic global search method for solving optimization problems. The genetic algorithm creates a population and applies genetic operators such as mutation and crossover for finding the best solution. The three most important requirements for using the genetic algorithms are the definition of an objective function, the implementation of a genetic representation, and the implementation of genetic operators. The genetic algorithm is an iterative scheme where the population is modified using the best features of the 'genes' from previous generations. The selection, crossover and mutation operators are applied to identify the best solution[6, 12, 13].

Pattern search is an optimization process also known as the direct-search, derivative-free and black-box method. It can be used on problems that are not continuous or differentiable. It was named by Hooke and Jeeves in 1961 and does not require an optimized function gradient. Fermi and Metropolis at the Los Alamos nuclear laboratory later developed a more advanced method with an adjustable mesh size. Pattern search evaluates the objective function at the lattice point centered on the current iteration to find the best objective function value then defines a new iteration at the resulting lattice point. The algorithm also expands or contracts the lattice depending on whether a more appropriate solution has been found. The generation point 
in a pattern is determined by polling, with the maximum number of points generated at each step. The efficiency of the solution can be improved by controlling the polling order of the points[3, 12, 14].

\section{Circular Restricted Three Body Problem}

The general three body problem posits that three bodies with arbitrary masses attract one another according to the universal law of gravitation. The gravitational three body problem requires 18 integrals of motion, but the number of integrals could be reduced to 8 by using conserved quantities; six of these scalar integrals are determined from the conservation of linear momentum, three from the conservation of total angular momentum, and one integral from the conservation of energy[15].

The three body problem can be simplified into a circular restricted three body problem defined as a system of two bodies in a circular orbit about their barycenters, and a third body with negligible mass. In the Earth-Moon barycentric frame, the barycenter of the two celestial bodies becomes the origin. The $\mathrm{x}$ axis is parallel to the line between the Earth and Moon, and is directed from the Earth toward the Moon. The $\mathrm{y}$ axis is 90 degrees from the $\mathrm{x}$ axis in the Earth and Moon's plane of motion. The $y$ axis is positive in the general direction of the motion of the Moon relative to the Earth. The $\mathrm{z}$ axis is defined to complete a right-handed coordinate system and is normal to the plane of motion spanned by the $\mathrm{x}$ and $y$ axes[16]. From a combination of the kinematic expansion, a scalar, second order and nonlinear set of differential equations are derived as:

$$
\begin{aligned}
& \ddot{x}=2 \dot{y}+x-\frac{(1-\mu)(x+\mu)}{d^{3}}-\frac{\mu(x-1+\mu)}{r^{3}}+\frac{F_{x}}{m} \\
& \ddot{y}=-2 \dot{x}+y-\frac{(1-\mu) y}{d^{3}}-\frac{\mu y}{r^{3}}+\frac{F_{y}}{m} \\
& \ddot{z}=-\frac{(1-\mu) z}{d^{3}}-\frac{\mu z}{r^{3}}+\frac{F_{z}}{m}
\end{aligned}
$$

where $\mathrm{d}$ and $\mathrm{r}$ denote the non-dimensional distance from the Earth to the spacecraft and the non-dimensional distance from the Moon to the spacecraft, respectively. $\mu$ and $(1-\mu)$ represent the non-dimensional mass of the Moon and the non-dimensional mass of the Earth, respectively. $F_{x}, F_{y}$ and $F_{z}$ denote external perturbation forces on the $\mathrm{x}$ and $\mathrm{y}$ axes and the thrust of the spacecraft respectively.

On one of the three collinear liberation points $\left(\mathrm{x}_{\mathrm{e}}\right)$ in the planar case, it is assumed that the external perturbation and thrust are zero. Then, Eqs.(1) (3) can be written as:

$$
\ddot{x}=2 \dot{y}+(2 c+1) x
$$

$$
\begin{aligned}
& \ddot{y}=-2 \dot{x}-(c-1) y \\
& \ddot{z}=-c z
\end{aligned}
$$

where $c=\frac{(1-\mu)}{\left|x_{\mathrm{e}}+\mu\right|^{3}}+\frac{\mu}{\left|\mathrm{x}_{\mathrm{e}}-1+\mu\right|^{3}}$.

The eigenvalue of Eqs.(4) (6) is

$$
\begin{aligned}
& \lambda_{1,2}= \pm \sqrt{-\beta_{1}+\sqrt{\beta_{1}^{2}+\beta_{2}^{2}}} \\
& \lambda_{3,4}= \pm \mathrm{i} \sqrt{\beta_{1}+\sqrt{\beta_{1}^{2}+\beta_{2}^{2}}} \\
& \lambda_{5,6}= \pm \mathrm{i} \sqrt{\mathrm{c}}
\end{aligned}
$$

where $\beta_{1}=2-\frac{c}{2}$ and $\beta_{2}=\sqrt{(2 c+1)(c-1)}$.

The general solution is written as:

$$
\begin{aligned}
& x(t)=\alpha_{1} e^{\lambda t}+\alpha_{2} e^{-\lambda t}+A_{x} \cos \left(\omega_{x y} t+\varphi\right) \\
& y(t)=-k_{1} \alpha_{1} e^{\lambda t}+k_{2} \alpha_{2} e^{-\lambda t}-k_{2} A_{x} \sin \left(\omega_{x y} t+\varphi\right) \\
& z(t)=A_{z} \cos \left(\omega_{z} t+\psi\right)
\end{aligned}
$$

where $\mathrm{k}_{1}=\frac{2 \mathrm{c}+1-\omega_{\mathrm{z}}^{2}}{2 \omega_{\mathrm{z}}}, \mathrm{k}_{2}=\frac{2 \mathrm{c}+1+\omega_{\mathrm{xy}}^{2}}{2 \omega_{\mathrm{xy}}} \cdot \alpha_{1}, \alpha_{2}, \mathrm{~A}_{\mathrm{x}}$ and $\mathrm{A}_{\mathrm{z}}$ can be calculated from the initial condition, and $\varphi$ and $\psi$ are angular phases.

To analyze the periodic motion, assume $\alpha_{1}$ and $\alpha_{2}$ are zero. Then the linearized solution becomes[15] :

$$
\begin{aligned}
& x(t)=A_{x} \cos \left(\omega_{x y} t+\varphi\right) \\
& y(t)=-k_{2} A_{x} \sin \left(\omega_{x y} t+\varphi\right) \\
& z(t)=A_{z} \cos \left(\omega_{z} t+\psi\right)
\end{aligned}
$$

The different types of periodic orbits near a collinear point are;

$\mathrm{A}_{\mathrm{x}} \neq 0$ \& $\mathrm{A}_{\mathrm{z}}=0$ : Planar Lyapunov Orbits

$\mathrm{A}_{\mathrm{x}}=0 \& \mathrm{~A}_{\mathrm{z}} \neq 0$ : Vertical Lyapunov Orbits

$A_{x} \neq 0 \& A_{z} \neq 0 \& \omega_{x y} \neq \omega_{z}$ : Lissajous Orbits(or Quasi-Halo Orbits)

$A_{x} \neq 0$ \& $A_{z} \neq 0$ \& $\omega_{x y}=\omega_{z}$ : Halo Orbits

The Lyapunov orbits are divided into a horizontal Lyapunov orbit and a vertical Lyapunov orbit. The horizontal Lyapunov orbit is a planar orbit with no out-of-plane motion and lies entirely within the orbital plane of the primaries. The vertical Lyapunov orbit is almost a vertical orbit with a typical eight-shape doubly symmetric geometry which is axisymmetric about the $\mathrm{x}$-axis and symmetric with respect to the xz plane.

The Lissajous orbit is defined as a trajectory with an 
in-plane and out-of-plane oscillation, but the oscillation frequencies of the in-plane and out-of-plane motion differ over time. The Lissajous orbits can be seen as quasisymmetric to the xy-plane and xz-plane.

The halo orbit has an in-plane and out-of-plane motion. However, the frequency stays the same and the orbit is periodic. The symmetricity to the xy-plane of the primaries no longer exists. The halo orbit only exists for the minimum in-plane amplitude and provides an exclusion zone located around the line connecting the primaries.

The quasi-halo orbit is a "mixture" of the Lissajous and halo orbits. The orbit circulates through the same path as the halo orbit, but its shape is similar to torus. The quasihalo orbit originates from the Lissajous orbits with a specific minimum boundary value of the out-of-plane amplitude. At this boundary amplitude, the Lissajous orbit loses their symmetry with respect to the xy-plane and starts to develop an exclusion zone about the line connecting the primaries. It is very useful for science and observation missions with formation flights[3, 17, 18].

\section{Insertion Maneuver Planning for Earth- Moon L2 Quasi-Halo Orbit}

In CR3BP (Circular Restricted Three Body Problem), the third small body is located at the Lagrange point where the gravitational pull of two large bodies is balanced. There exist five Lagrange points in the Earth-Moon rotating coordinate system. L1, L2 and L3 are saddle points which are stable along the axis between the two primary bodies but unstable in the other direction. Station keeping maneuvers are therefore required to remain at these points. The L4 and L5 points are stable and do not require station keeping maneuvers. The Quasi-halo orbit can perform periodic motion but is not actually an orbit around the Lagrange point. The Quasi-halo orbit can also supply trajectories to transfer into and out of the Lagrange orbits by providing low energy manifolds[18, 19].

A spacecraft placed in an Earth-Moon L2 quasi-halo orbit can communicate between the Earth and the far side of the Moon. This quasi-halo orbit could be used to establish a lunar space station that can serve as a gateway for solar system exploration or as a fueling spacecraft to help other spacecrafts depart the Earth Moon system with lower maneuver costs. This can reduce the overall size of the spacecraft as well as the cost of the mission.

For this mission, a spacecraft requires an impulsive burn to transfer from the Earth parking orbit to the point of insertion into the quasi-halo orbit. Additionally, an insertion maneuver is required to approach an Earth-
Moon L2 quasi-halo orbit. There are three types of transfers to enter the quasi-halo orbit; direct transfer, low thrust transfer and ballistic lunar trajectory transfer. Parker[20] calculated the fuel savings for these different types of transfers for a spacecraft travelling from the Earth parking orbit at $185 \mathrm{~km}$ altitude to the Earth-Moon L2 point. Parker showed that the direct transfer from LEO to L2 requires a total delta- $\mathrm{V}$ of $3.9823 \mathrm{~km} / \mathrm{s}$ over 5 days of transfer time. This paper adopted the direct transfer because the low thrust and ballistic lunar trajectory transfers to the Moon require relatively long transfer times. Such long durations to reach the quasi-halo orbit would decrease the reliability of the mission[21].

Although halo orbit is both periodic and time independent in the CR3BP, it does not consider all the gravitational and non-gravitational effects of the full solar system, the solar radiation pressure and the non-uniform gravity of the Earth and the Moon. A differential correction method is used to compute maneuver time and delta- $\mathrm{V}$ to inject a satellite into an Earth-Moon L2 quasi-halo orbit departing from a circular low Earth parking orbit. A quasi-halo orbit insertion maneuver is also investigated using the differential corrector. The STK/Astrogator contains full-force models and interplanetary propagation/targeting techniques. Table 2 lists the initial state of a spacecraft on the low Earth orbit.

The mission starts from an initial low Earth circular parking orbit followed by a quasi-halo orbit injection maneuver near the Earth-Moon L2 Lagrange point. The insertion point of the quasi-halo orbit is selected at $20,000 \mathrm{~km}$ in the $\mathrm{z}$ axis, and $0 \mathrm{~km}$ in the $\mathrm{x}$ and $\mathrm{y}$ axes with the origin of the EarthMoon L2 point. To make a periodic orbit for station keeping, the end condition for this propagation segment after one period requires that the spacecraft cross the Earth-Moon L2 Z-X plane with $\mathrm{Vx}=0$. The delta-v required for quasi-halo orbit insertion using the differential-corrector method in STK Astrogator is obtained as shown in Table 3. Table 3 lists the maneuver information and state of the spacecraft on transfer orbit for insertion into the Earth-Moon L2 quasihalo orbit[22].

After the insertion maneuver into the quasi-halo orbit, it is assumed that collision is expected from an uncontrolled object on the Earth-Moon L2 quasi-halo orbit. Table 4 shows the initial state of the uncontrolled object. If a potential collision risk is detected, a precise orbit should be determined by using all possible tracking data from global observation sites on Earth. Table 5 lists orbit determination covariance and the collision information of the assumed spacecraft with the uncontrolled object.

Once the collision risk is greater than a maximum threshold, a plan for avoidance maneuver should be generated. An 
acceptable avoidance maneuver plan should produce an orbit change that reduces the risk of collision while still meeting positioning constraints to retain the quasi-halo orbit.

Figure 2 shows a flowchart to calculate the optimal maneuver and evaluate its performance for collision avoidance while remaining in the quasi-halo orbit. STK/Pro to is used as the starting point to model, analyze, visualize and communicate with other components. The STK/ Astrogator propagates the orbit of a spacecraft with delta-V at the maneuver start time generated from the Matlab global optimization algorithm. It then integrates an accurate full perturbation model that includes solar radiation pressure and planet gravitational perturbation, where "Tman" and " $\triangle$ VRIC" represent the maneuver time in UT, the delta-V in radial, in-track and cross-track respectively. The collision probability is obtained from STK/AdvCAT, which adopts the Alfano formula[23, 24, 25, 26, 27]. Alfano developed the twodimensional collision probability equation in the encounter plane given in Cartesian space as a combination of error functions and exponential terms as shown in Eqs.(16) (18). Alfano used a rectangular coordinate system to obtain the collision probability value, but did not assume that the velocity remains constant for each tube segment.

$$
\begin{aligned}
& P_{c}=\frac{R_{o b j} \cdot 2}{\sqrt{8 \cdot \pi} \cdot \sigma_{x} \cdot n} \cdot(\alpha+\beta) \cdot \exp \left[\frac{-\left[\frac{R_{o b j} \cdot(2 \cdot i-n)}{n}+x_{m}\right]^{2}}{2 \cdot \sigma_{x}^{2}}\right] \\
& \alpha=\sum_{i=0}^{n} \operatorname{erf}\left[\frac{y_{m}+2 \cdot R_{o b j} / n \cdot \sqrt{(n-1) \cdot i}}{\sigma_{y} \sqrt{2}}\right] \\
& \beta=\sum_{i=0}^{n} \operatorname{erf}\left[\frac{-y_{m}+2 \cdot R_{o b j} / n \cdot \sqrt{(n-1) \cdot i}}{\sigma_{y} \sqrt{2}}\right]
\end{aligned}
$$

$\mathrm{R}_{\mathrm{obj}}$ denotes the total cross sectional radius of two objects assumed to be circular. $\sigma_{\mathrm{x}}, \sigma_{\mathrm{y}}, \mathrm{x}_{\mathrm{m}}$ and $\mathrm{y}_{\mathrm{m}}$ denote the standard deviation and distance from the center in the direction of each axis.

The control states are impulsive burn time and delta- $v$ in the radial, in-track and cross-track directions.

The fitness value can be written as

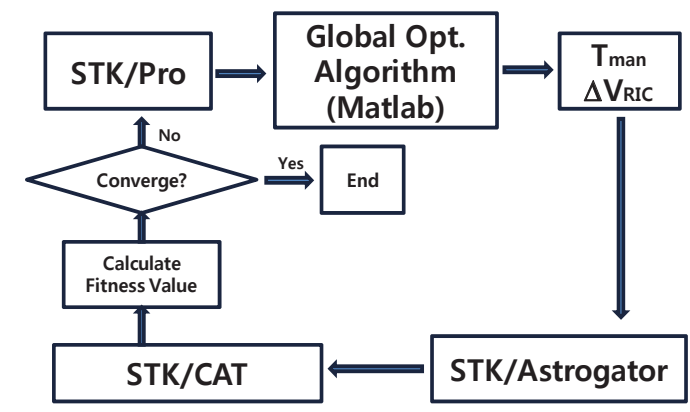

Fig. 2. A Flowchart of Optimal Maneuver Planning

$$
\mathrm{F}=\sum_{\mathrm{i}=1}^{\mathrm{N}} \Delta \mathrm{L}_{\mathrm{i}}\left(\mathrm{t}_{\mathrm{i}}\right)+\mathrm{KP}_{\mathrm{c}}
$$

where $\Delta \mathrm{L}_{\mathrm{i}}$ represents the ith position difference between the reference orbit and an orbit generated by the optimization algorithm. $\mathrm{P}_{\mathrm{c}}$ and K represent a maximum collision probability and a weighting factor, respectively. The maximum collision probability is set to $10^{-4}$ and collision miss distance is set to $100 \mathrm{~km}$.

\section{Simulation Results}

For comparison, ten simulation trials were performed to

\begin{tabular}{|c|c|}
\hline Item & Value \\
\hline Coordinate System & Earth Inertial \\
\hline Coordinate Type & Target Vector Outgoing Asymptote \\
\hline Orbit Epoch & 1 Jul 2025 12:00:00.000UTCG \\
\hline Radius of Periapsis & $6778 \mathrm{~km}$ \\
\hline C3 Energy & $-1.62335 \mathrm{~km}^{\wedge} 2 / \mathrm{Sec}^{\wedge} 2$ \\
\hline RA of Outgoing Asymptote & $252.107 \mathrm{deg}$ \\
\hline $\begin{array}{c}\text { Declination of Outgoing } \\
\text { Asymptote }\end{array}$ & $-24.848 \mathrm{deg}$ \\
\hline $\begin{array}{l}\text { Velocity Azimuth at } \\
\text { Periapsis }\end{array}$ & $82.9741 \mathrm{deg}$ \\
\hline True Anomaly & $0.000786134 \mathrm{deg}$ \\
\hline Spacecraft Properties & $\begin{array}{l}\text { Total Mass: } 800 \mathrm{~kg} \text { (Dry Mass: } 500 \mathrm{~kg} \text {, Fuel } \\
\text { Mass : } 300 \mathrm{~kg} \text { ) } \\
\text { Area /Mass Ratio: } 1.25 \mathrm{e}-9 \mathrm{~km}{ }^{\wedge} 2 / \mathrm{kg} \\
\text { Tank Pressure : } 5000 \text { Pa, Fuel Density: } 1000 \\
\text { kg/m^3 } \\
\text { Cr: } 2.0, \text { Cd: } 2.0, \text { Drag Area: } 1 \mathrm{~m}^{\wedge} 2 \text {, SPR Area: } \\
\quad 1 \mathrm{~m}^{\wedge} 2 \\
\text { Solar Radiation Pressure Area : } 20 \mathrm{~m}^{\wedge} 2 \\
\text { Radiation Pressure Coefficient }: 1.0\end{array}$ \\
\hline
\end{tabular}
determine the influence of the cost function on each global

Table 2. The Initial State of the Spacecraft on the Earth Orbit for Injection to Transfer Orbit

Table 3. Insertion Maneuver by Differential Corrector

\begin{tabular}{c|c}
\hline Item & Value \\
\hline $\begin{array}{c}\text { Maneuver Type } \\
\text { Engine Model } \\
\text { CoordinateSystem } \\
\text { Maneuver Time }\end{array}$ & $\begin{array}{c}\text { Impulsive } \\
\text { Earth Inertial }\end{array}$ \\
Delta-V(km/sec) & Vx:0.88709, Vy: -0.29449, Vz: -0.12307 \\
Estimated Fuel Used & $219.342 \mathrm{~kg}$ \\
Estimated Burn & $1290.61 \mathrm{sec}$ \\
Duration &
\end{tabular}


Table 4. The Initial State of an Uncontrolled Object

\begin{tabular}{c|c}
\hline Central Body & Moon \\
Orbit Epoch & 15 Jul 2025 17:19:14.361 UTCG \\
Coordinate Type & Cartesian \\
Coordinate System & J2000 \\
Propagator & HPOP \\
Position(km) & X: 50665.7, Y: 3519.95, Z: -14372.3 \\
Velocity $(\mathrm{km} / \mathrm{sec})$ & Vx: 0.028431, Vy: 0.34545, Vz: 0.75294
\end{tabular}

Table 5. Orbit Determination Covariance and Collision Information

\begin{tabular}{c|cccc}
\hline & $\mathrm{T}(\mathrm{km})$ & $\mathrm{C}(\mathrm{km})$ & $\mathrm{N}(\mathrm{km})$ & Size $(\mathrm{m})$ \\
\hline L2 Probe & 5 & 5 & 5 & 5 \\
HALO Object & 5 & 5 & 5 & 50 \\
Collision Exp. & \multicolumn{5}{|c}{ 15 Jul 2025 17: 19: 14} \\
$\quad$ Time \\
Minimum & \multicolumn{4}{|c}{0.534} \\
Dist.(km) & \multicolumn{5}{|c}{$1.02 \mathrm{e}-2$} \\
Collision Prob. & \multicolumn{5}{c}{. }
\end{tabular}

optimization case. In this simulation, the optimization parameters 'MaxIter', 'MaxFunEvals' and 'TolFun' is set to 4000, 4000 and 10e-6, respectively. If the optimality measure is less than 'TolFun', the iterations end, and 'TolFun' can also be a relative bound. 'MaxIter' is a bound on the number of solver iterations. 'MaxFunEvals' is a bound on the number of function evaluations.

Figure 3.a c show the 3D view for one of 10 cases in view of the Moon fixed frame to get an insertion maneuver into a quasi-halo orbit by a simulated annealing, a genetic algorithm and a pattern search algorithm. The uncontrolled object approaches from the direction of the Moon toward the spacecraft on the quasi-halo orbit. Possible collision is declared by the conjunction analysis software and the global optimal algorithm attempts to find the best solution with the boundary constraints provided. The fitness values are checked at each iteration. The white line indicates the Moon trajectory and the green line stands for the spacecraft trajectory before the maneuver for quasi-halo orbit insertion. The red line represents the quasi-halo orbit after the insertion maneuver converged using the global optimization algorithm. The quasi-halo orbit is periodic in the $\mathrm{Y}-\mathrm{Z}$ axis as shown in the left side images of Figs. 3.

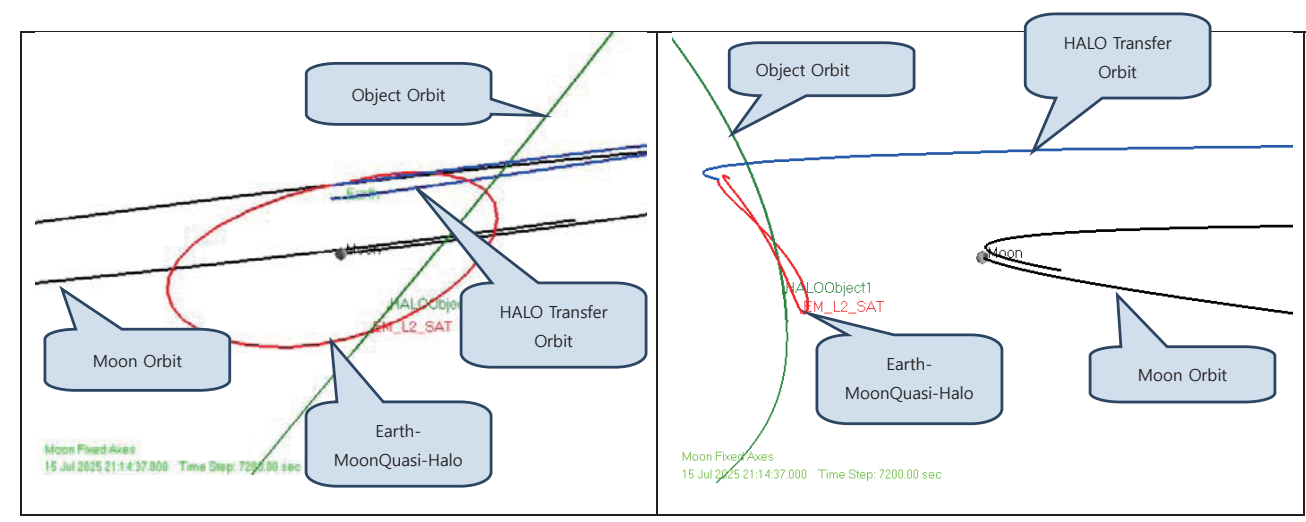

Fig. 3a. Simulation Results (Simulated Annealing Case 1)

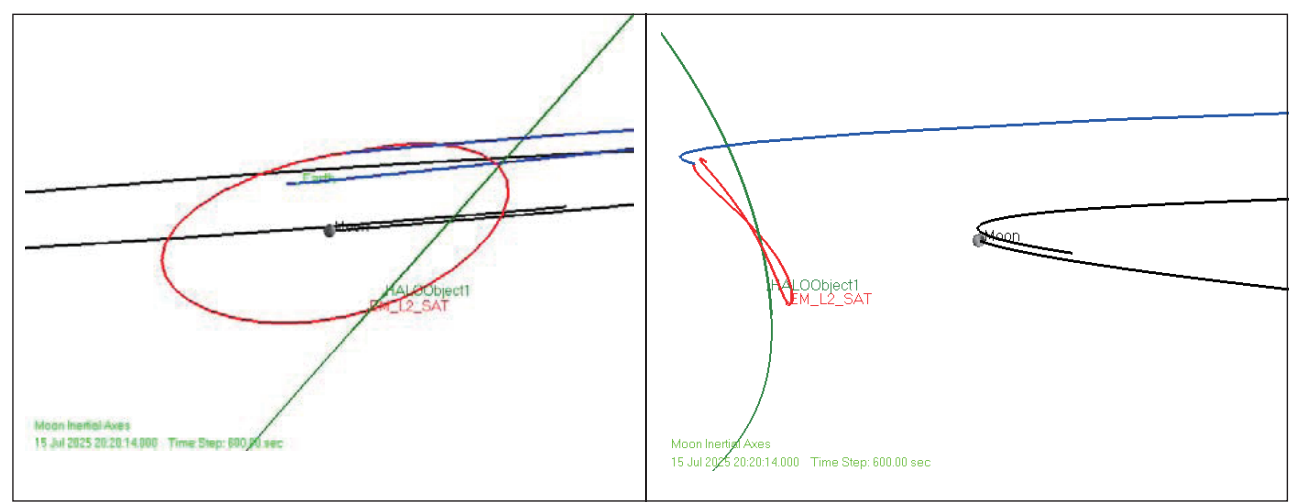

Fig. 3b. Simulation Results (Genetic Algorithm Case 1) 
The right side images of Figs. 3. in the $\mathrm{X}-\mathrm{Z}$ axis illustrate that inclinations are formed. Figs. 4 show radial, in-track and cross-track distance difference between the spacecraft trajectory generated by the differential corrector and the spacecraft trajectory generated by global optimization methods for the first case 1 .

Tables $6 \mathrm{a} \sim \mathrm{c}$ and Fig. 5a c show the simulation results for avoidance maneuver planning using global optimization.

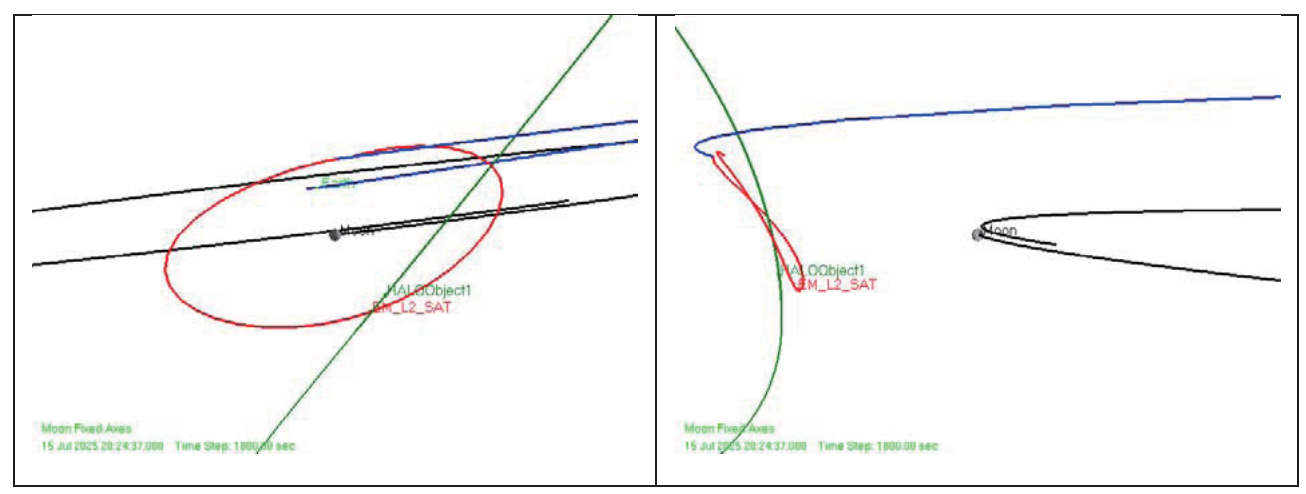

Fig. 3c. Simulation Results (Pattern Search Case 1)

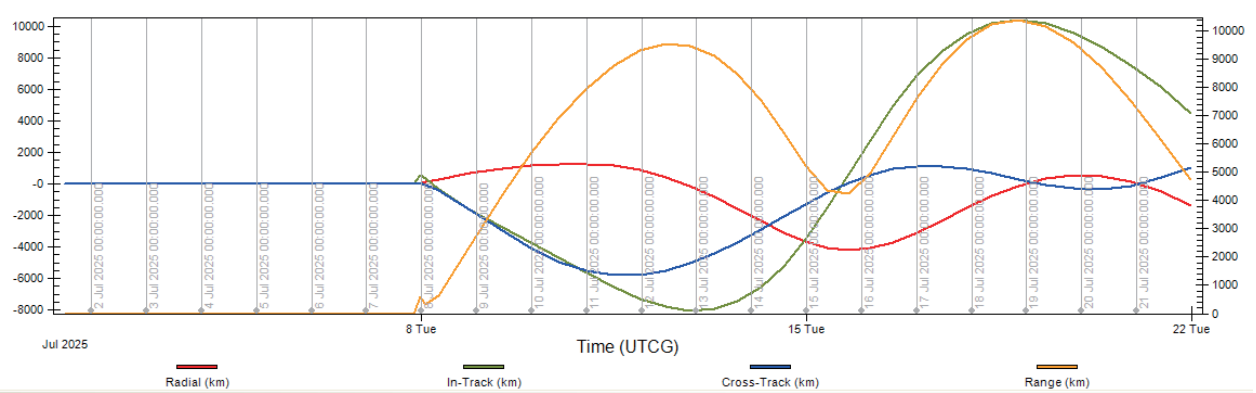

Fig. 4a. RIC and Range Distance Difference (Simulated Annealing Case 1)

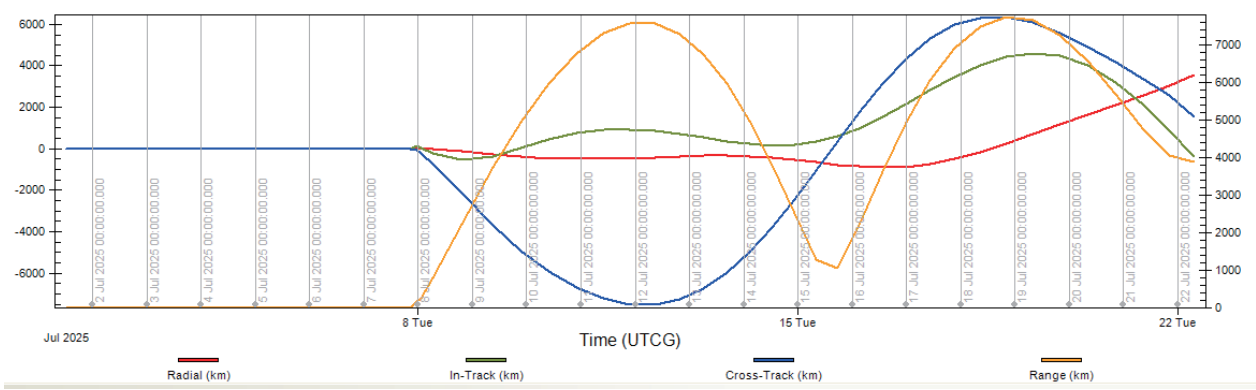

Fig. 4b. RIC and Range Distance Difference (Genetic Algorithm Case 1)

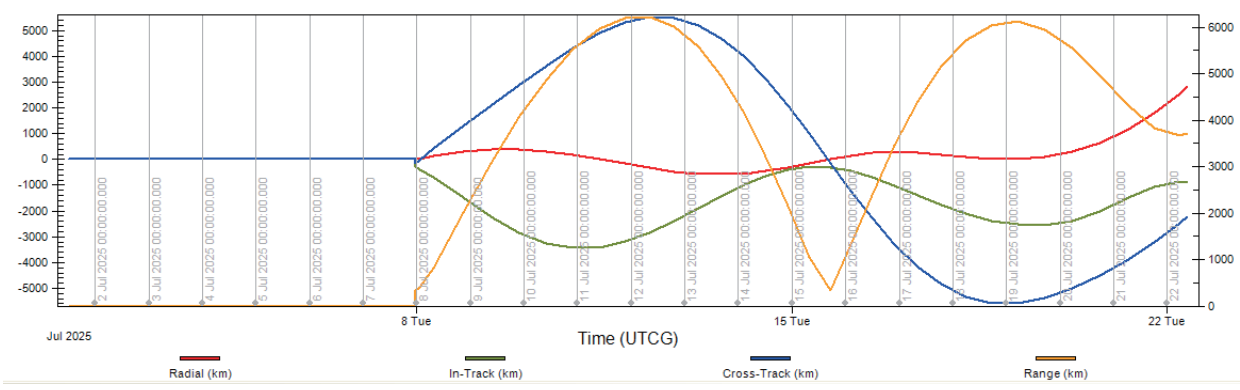

Fig. 4c. RIC and Range Distance Difference (Pattern Search Case 1) 
Sang-Cherl Lee Comparison of Global Optimization Methods for Insertion Maneuver into Earth-Moon L2 ...

Table 6a. Simulation Result after Avoidance Maneuver Planning Using Simulated Annealing

\begin{tabular}{|c|c|c|c|c|c|c|c|c|}
\hline $\begin{array}{l}\text { Case } \\
\text { No }\end{array}$ & $\begin{array}{l}\text { Man_Time } \\
\text { (UTCG) }\end{array}$ & $\begin{array}{c}\text { DelV_x } \\
(\mathrm{km} / \mathrm{s}, \mathrm{ECI})\end{array}$ & $\begin{array}{c}\text { DelV_y } \\
(\mathrm{km} / \mathrm{s}, \mathrm{ECI})\end{array}$ & $\begin{array}{c}\text { DelV_z } \\
(\mathrm{km} / \mathrm{s}, \mathrm{ECI})\end{array}$ & TotalDelV & Cost Fun & $\begin{array}{l}\text { Max. } \\
\text { Func. } \\
\text { Eval. }\end{array}$ & $\begin{array}{c}\text { Collision } \\
\text { Prob. }\end{array}$ \\
\hline 1 & 7 Jul 2025 23:10:01.920 & 0.850505 & -0.291555 & -0.130624 & 0.908530 & $3.2527 \mathrm{e} 4$ & 4000 & No Warn. \\
\hline 2 & 7 Jul 2025 23:10:01.920 & 0.901398 & -0.289530 & -0.131955 & 0.955907 & $1.7037 \mathrm{e} 4$ & 4000 & No Warn. \\
\hline 3 & 7 Jul 2025 23:10:01.920 & 0.889694 & -0.303757 & -0.097427 & 0.945155 & $1.6704 \mathrm{e} 4$ & 4000 & No Warn. \\
\hline 4 & 7 Jul 2025 23:10:01.920 & 0.864243 & -0.291628 & -0.130641 & 0.921428 & $2.4393 \mathrm{e} 4$ & 4000 & No Warn. \\
\hline 5 & 7 Jul 2025 23:28:19.200 & 0.865691 & -0.296707 & -0.119394 & 0.922882 & $2.3530 \mathrm{e} 4$ & 4000 & No Warn. \\
\hline 6 & 7 Jul 2025 23:29:36.960 & 0.838590 & -0.287033 & -0.140430 & 0.897409 & $3.7957 \mathrm{e} 4$ & 4000 & No Warn. \\
\hline 7 & 7 Jul 2025 23:19:06.240 & 0.837496 & -0.297909 & -0.114908 & 0.896300 & $4.2240 \mathrm{e} 4$ & 4000 & No Warn. \\
\hline 8 & 7 Jul 2025 23:10:27.840 & 0.921033 & -0.301070 & -0.097284 & 0.973863 & $3.4012 \mathrm{e} 4$ & 4000 & No Warn. \\
\hline 9 & 7 Jul 2025 23:23:25.440 & 0.826213 & -0.279156 & -0.154978 & 0.885762 & $4.8500 \mathrm{e} 4$ & 4000 & No Warn. \\
\hline 10 & 7 Jul 2025 23:20:24.000 & 0.869968 & -0.267055 & -0.179721 & 0.927611 & $3.4286 \mathrm{e} 4$ & 4000 & No Warn. \\
\hline
\end{tabular}

Table 6b. Simulation Result after Avoidance Maneuver Planning Using Genetic Algorithm

\begin{tabular}{|c|c|c|c|c|c|c|c|c|}
\hline Case & $\begin{array}{c}\text { Man_Time } \\
\text { (UTCG) }\end{array}$ & $\begin{array}{c}\text { DelV_x } \\
(\mathrm{km} / \mathrm{s}, \mathrm{ECI})\end{array}$ & $\begin{array}{c}\text { DelV_y } \\
(\mathrm{km} / \mathrm{s}, \mathrm{ECI})\end{array}$ & $\begin{array}{c}\text { DelV_z } \\
(\mathrm{km} / \mathrm{s}, \mathrm{ECI})\end{array}$ & Total DelV & $\begin{array}{c}\text { Best } \\
\text { Cost Fun }\end{array}$ & $\begin{array}{l}\text { Max. } \\
\text { Fun. } \\
\text { Eval. }\end{array}$ & $\begin{array}{c}\text { Collision } \\
\text { Prob. }\end{array}$ \\
\hline 1 & 7 Jul 2025 23:23:34.080 & 0.897378 & -0.304766 & -0.094120 & 0.952380 & $2.902 \mathrm{e} 4$ & 4000 & No Warn. \\
\hline 2 & 7 Jul 2025 23:28:36.480 & 0.884162 & -0.287613 & -0.139542 & 0.940179 & $1.353 \mathrm{e} 4$ & 4000 & No Warn. \\
\hline 3 & 7 Jul 2025 23:20:15.360 & 0.879809 & -0.285391 & -0.144266 & 0.936123 & $1.621 \mathrm{e} 4$ & 4000 & No Warn. \\
\hline 4 & 7 Jul 2025 23:10:27.840 & 0.909170 & -0.296791 & -0.112597 & 0.962992 & $2.455 \mathrm{e} 4$ & 4000 & No Warn. \\
\hline 5 & 7 Jul 2025 23:08:26.880 & 0.920401 & -0.293585 & -0.117621 & 0.973224 & $2.705 \mathrm{e} 4$ & 4000 & No Warn. \\
\hline 6 & 7 Jul 2025 23:25:09.120 & 0.888628 & -0.294739 & -0.122487 & 0.944211 & $1.373 \mathrm{e} 4$ & 4000 & No Warn. \\
\hline 7 & 7 Jul 2025 23:25:00.480 & 0.879775 & -0.296022 & -0.120297 & 0.936005 & $1.855 \mathrm{e} 4$ & 4000 & No Warn. \\
\hline 8 & 7 Jul 2025 23:28:36.480 & 0.869536 & -0.293840 & -0.126260 & 0.926486 & $2.019 \mathrm{e} 4$ & 4000 & No Warn. \\
\hline 9 & 7 Jul 2025 23:13:55.200 & 0.917336 & -0.285886 & -0.137184 & 0.970596 & $2.292 \mathrm{e} 4$ & 4000 & No Warn. \\
\hline 10 & 7 Jul 2025 23:17:48.480 & 0.909015 & -0.288382 & -0.133660 & 0.962984 & $1.891 \mathrm{e} 4$ & 4000 & No Warn. \\
\hline
\end{tabular}

Table 6c. Simulation Result after Avoidance Maneuver Planning Using Pattern Search

\begin{tabular}{|c|c|c|c|c|c|c|c|c|}
\hline Case & $\begin{array}{l}\text { Man_Time } \\
\text { (UTCG) }\end{array}$ & $\begin{array}{c}\text { DelV_x } \\
(\mathrm{km} / \mathrm{s}, \mathrm{ECI})\end{array}$ & $\begin{array}{c}\text { DelV_y } \\
(\mathrm{km} / \mathrm{s}, \mathrm{ECI})\end{array}$ & $\begin{array}{c}\text { DelV_z } \\
(\mathrm{km} / \mathrm{s}, \mathrm{ECI})\end{array}$ & Total DelV & $\begin{array}{l}\text { Best Cost } \\
\text { Fun }\end{array}$ & $\begin{array}{l}\text { Max. } \\
\text { Func } \\
\text { Eval. }\end{array}$ & $\begin{array}{c}\text { Collision } \\
\text { Prob. }\end{array}$ \\
\hline 1 & 7 Jul 2025 23:14:21.120 & 0.886258 & -0.283714 & -0.147013 & 0.942104 & $1.6527 \mathrm{e} 4$ & 279 & No Warn. \\
\hline 2 & 7 Jul 2025 23:11:36.960 & 0.902937 & -0.296465 & -0.114899 & 0.957282 & $2.1010 \mathrm{e} 4$ & 211 & No Warn. \\
\hline 3 & 7 Jul 2025 23:19:58.080 & 0.917523 & -0.298591 & -0.106289 & 0.970722 & $2.8470 \mathrm{e} 4$ & 125 & No Warn. \\
\hline 4 & 7 Jul 2025 23:19:58.080 & 0.919001 & -0.297857 & -0.107845 & 0.972067 & $2.8472 \mathrm{e} 4$ & 414 & No Warn. \\
\hline 5 & 7 Jul 2025 23:20:24.000 & 0.890843 & -0.295652 & -0.119651 & 0.946217 & $1.5324 \mathrm{e} 4$ & 896 & No Warn. \\
\hline 6 & 7 Jul 2025 23:14:21.120 & 0.899524 & -0.282099 & -0.148789 & 0.954391 & $1.8371 \mathrm{e} 4$ & 112 & No Warn. \\
\hline 7 & 7 Jul 2025 23:19:58.080 & 0.915705 & -0.293800 & -0.119325 & 0.969058 & $2.3589 \mathrm{e} 4$ & 508 & No Warn. \\
\hline 8 & 7 Jul 2025 23:12:54.720 & 0.886717 & -0.283660 & -0.147006 & 0.942519 & $1.6693 \mathrm{e} 4$ & 278 & No Warn. \\
\hline 9 & 7 Jul 2025 23:19:58.080 & 0.899802 & -0.298733 & -0.110234 & 0.954482 & $2.1358 \mathrm{e} 4$ & 119 & No Warn. \\
\hline 10 & 7 Jul 2025 23:19:58.080 & 0.915109 & -0.292987 & -0.121500 & 0.968519 & $2.2820 \mathrm{e} 4$ & 152 & No Warn. \\
\hline
\end{tabular}


The maximum iteration for each optimization method was set to 4,000 . For the simulated annealing, the minimum bestcost-function was $1.6704 \mathrm{e} 4$, while the maximum best-costfunction was $4.8500 \mathrm{e} 4$. The minimum best-cost-function was $1.353 \mathrm{e} 4$ using the genetic algorithm, and the maximum was $2.902 \mathrm{e} 4$. With the pattern search method, the minimum best-cost-function was $1.53249 \mathrm{e} 4$, and the maximum was

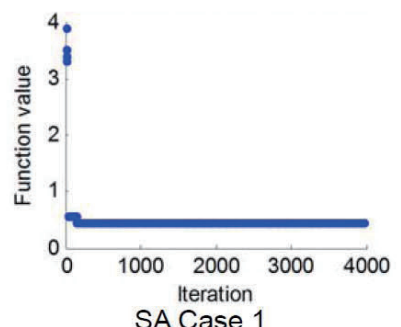

SA Case 1
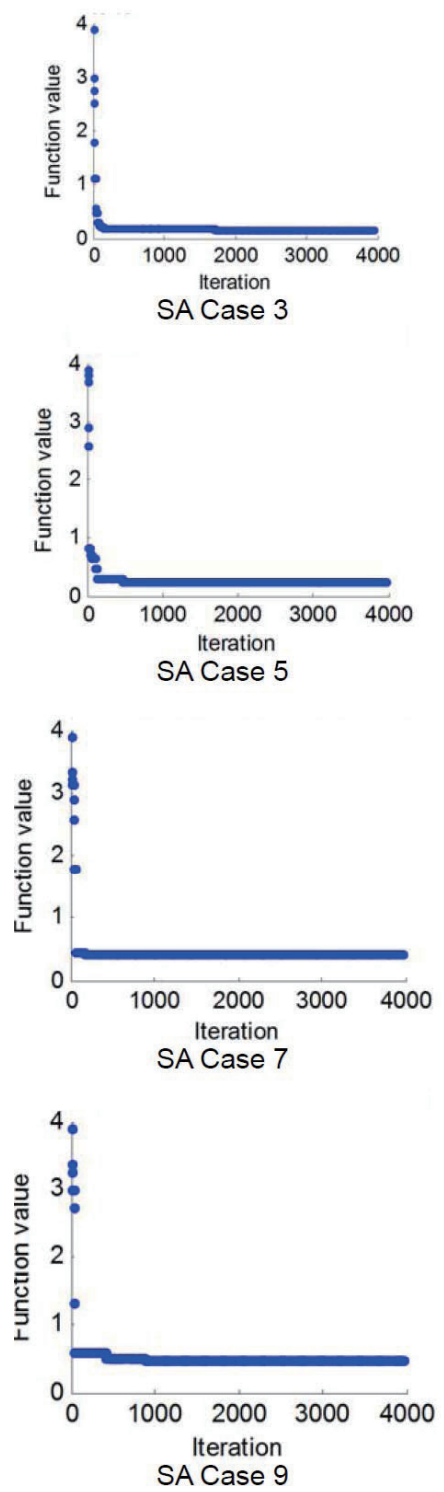

Fig. 5a. Fitness Function by Simulated Annealing 2.8472e4. All cases produce periodic orbits. However, the pattern search converged faster than the others. Before avoidance maneuver, the predicted distance reached approximately $0.533 \mathrm{~km}$ and the collision probability was 1.02e-2. After collision avoidance, all of the cases detected no collision because the collision warning is set to $100 \mathrm{~km}$ as the miss distance.
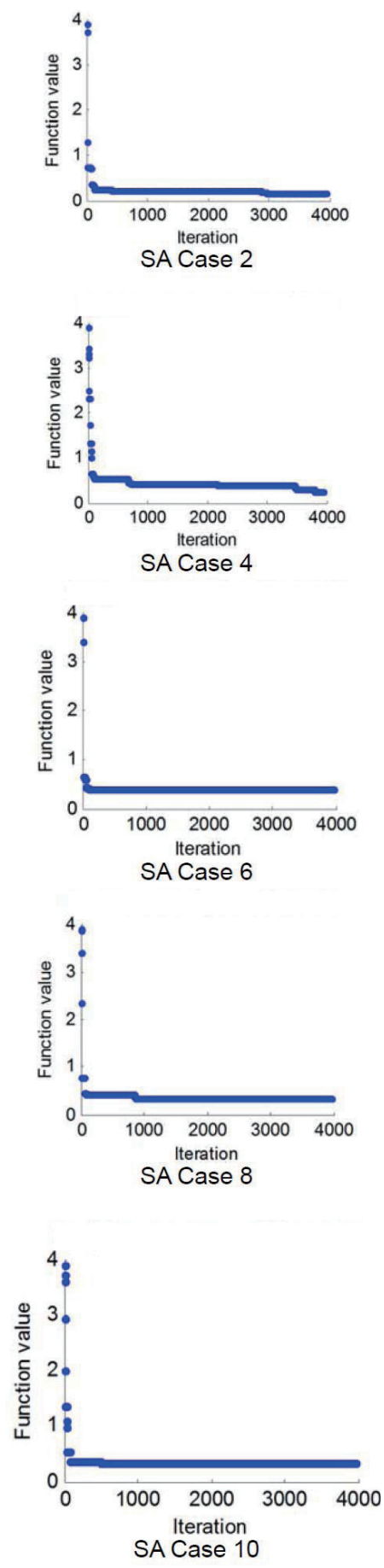
Figure 5.a c show the best fitness function values for all iterations. The fitness function of the simulated annealing method decreased faster than the other cases, but it ended at the maximum iteration value. The minimum and maximum fitness function values of the genetic algorithm and the pattern search are similar, though the pattern search values converged more rapidly. The pattern search method
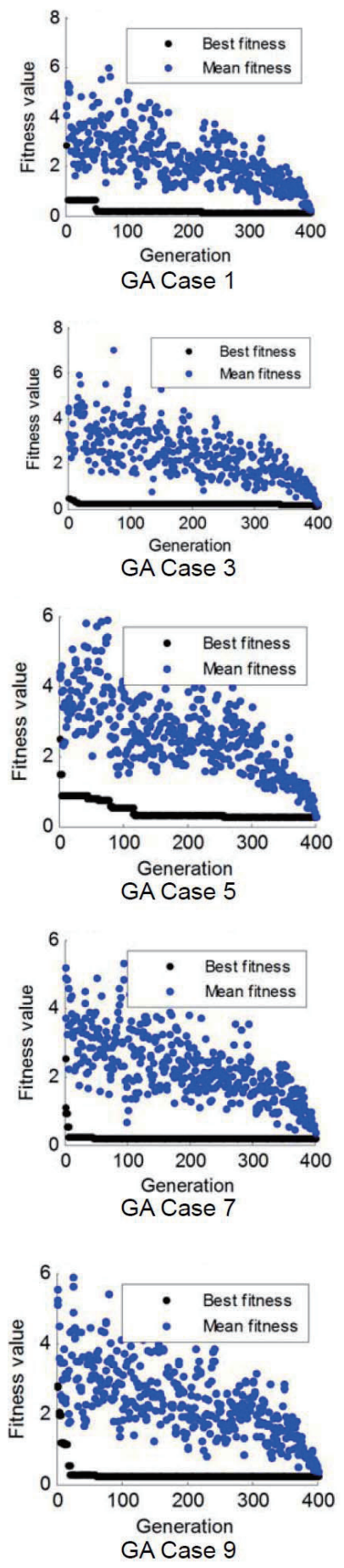

Fig. 5b. Fitness Function by Genetic Algorithm converged more rapidly than the other cases but ended at the maximum 1000 iterations.

\section{Conclusion}

This study investigated an insertion maneuver considering collision avoidance against an uncontrolled
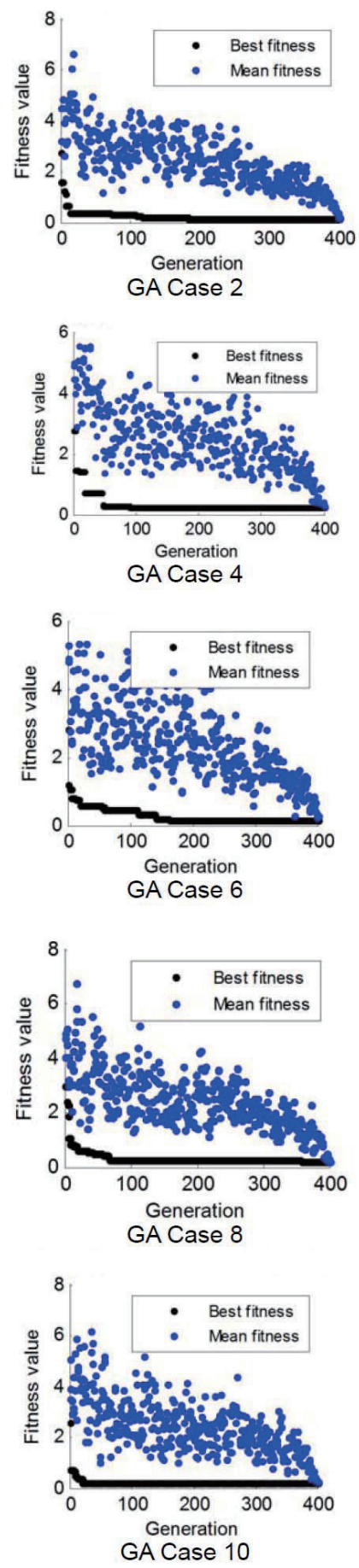

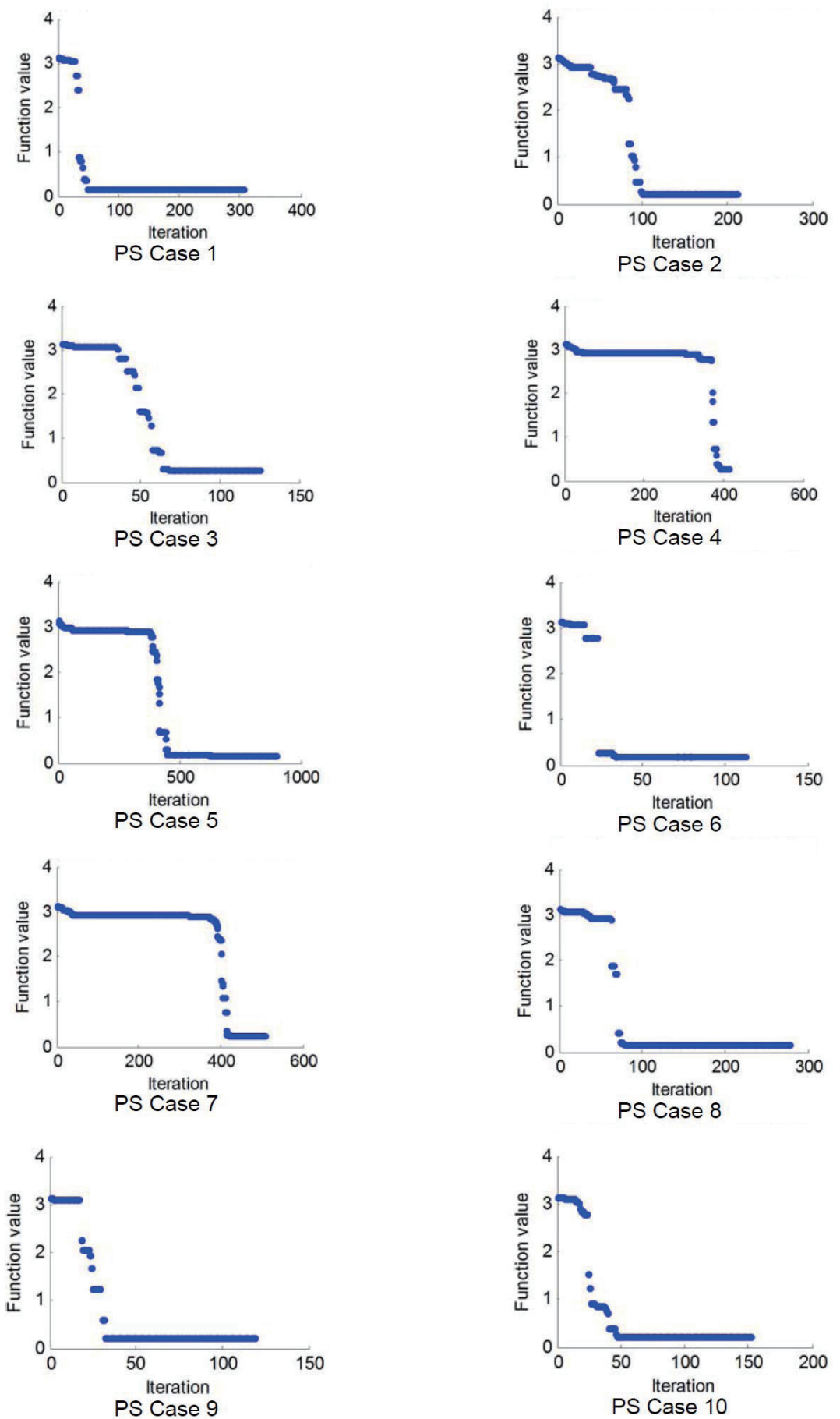

Fig. 5c. Fitness Function by Pattern Search

object in the Earth-Moon L2 quasi-halo orbit. Global optimization algorithms including simulated annealing, genetic algorithm and pattern search were used to minimize the differences between a reference orbit and an orbit after avoidance maneuver. The simulations were performed using the STK/Astrogator and Matlab global optimization toolbox. All three algorithms are used to obtain the maneuver start time and delta-V so as to reduce the collision probability from uncontrolled objects and to retain the reference trajectory calculated by the differential corrector with the 
STK/Astrogator.

Thegeneticalgorithmmethod could createnewindividuals by combining different solutions, while simulated annealing created a new individual by modifying only a solution with a local move. Consequently, the genetic algorithm method performed better than the simulated annealing method.

From the performance point of view, genetic algorithm is a slow starter with more accuracy, while the simulated annealing method starts quickly and can obtain a relatively good solution in a short time. The convergence of the genetic algorithm took longer than that of the simulated annealing method.

The genetic algorithm method uses a large search space and requires a lot of iterations for optimization, while the pattern search method finds a certain direction and requires relatively fewer steps for solving the problem. Additionally, the genetic algorithm method optimizes its search space in complicated problems, while the pattern search method displays the best performance with a relatively smaller search space and less computing time.

This approach can be applied to an actual quasi-halo orbit mission for inserting a spacecraft into a halo orbit with collision avoidance maneuver for the Earth-Moon and the Sun-Earth Lagrange point mission.

\section{Acknowledgements}

The research was supported by the "Electro-optic Space Surveillance System" of the National Agenda Project funded by the Korea Research Council of Fundamental Science \& Technology. Authors would like to thank KRCFS and KARI for their support.

\section{References}

[1] Dawn Perry Gordon., Transfers to Earth-Moon L2 HALO Orbits Using Lunar Proximity and Invariant Manifolds, M.S. Thesis, Purdue University, August 2008.

[2] Bernelli F, Topputo F. and Massari M., Assessment of Mission Design Including Utilization of Libration Points and Weak Stability Boundaries, Final Report, ESTEC Contract No. 18147/04/NL/MV, 2004.

[3] http://en.wikipedia.org/wiki/Lagrangian_point

[4] Woodard, M., Folta, D., and Woodfork, D., "ARTEMIS: The First Mission to the Lunar Libration Points", presented at the 21st International Symposium on Space Flight Dynamics (ISSFD), Toulouse, France, September 28 - October 2, 2009.

[5] http://www.answers.com/topic/space-exploration
[6] dos Santos, D.P.S., and Prado, A.F.B.A., Minimum Fuel Multi-Impulsive Orbital Maneuvers Using Genetic Algorithms, IAA-AAS-DyCoSS1-11-01.

[7] Michael A. Paluszek, and Stephanie J. Thomas, “Trajectory Optimization Using Global Methods", The 29th International Electric Propulsion Conference Princeton University, October 31 - November 4, 2005.

[8] Lu-sha Wang, Zhi-gang Wang, and Wei Li, Optimization of Parameters Injecting into Orbits Based on the Pattern Search Method, 2009 First International Conference on Information Science and Engineering(ICISE), 2009, pp.38233826.

[9] Willem H. de Vries, Maneuver Optimization through Simulated Annealing, Lawrence Livermore National Laboratory, LLNL-CONF-497728.

[10] Hae-Dong Kim, and Eun-Sum Sim, "Lunar Transfer Trajectory Design Using a WSB", Proceeding of the 2009 KSAS Fall Conference, 2009. 21-1201.

[11] Michael A. Paluszek, and Stephanie J. Thomas, “Trajectory Optimization Using Global Methods", The 29th International Electric Propulsion Conference Princeton University, October 31 - November 4, 2005.

[12] MATLAB, Global Optimization ToolBox, Mathworks.

[13] Sang-Cherl Lee, Hae-Dong Kim, and Jinyoung Suk, "Collision Avoidance Maneuver Planning Using GA for LEO and GEO Satellite Maintained in Keeping Area", Int'l J. of Aeronautical \& Space Sci., Vol. 13, No.4, 2012, pp. 474-483.

[14] Magnus Erik Hvass Pedersen, Tuning \& Simplifying Heuristical Optimization, Ph.D. Thesis for the degree of Doctor of Philosophy, January 2010

[15] Rosário Laureano, Determinism Versus Predictability in the Context of Poincare's Work on the Restricted 3-Body Problem, University Institute of Lisbon, Portugal, Vol. 2, No. 2, March 2012.ISSN-L: 2223-9553, ISSN: 2223-9944.

[16] Pierpaolo Pergola, Chiara Finocchietti, "Circular Restricted Tree Body Model”, Space System Lecture, Pisa, November 2012.

[17] F.Renk, M.Landgraf, "Sun-Earth Libration point transfer options with intermediate HEO", ActaAstronautica, Vol. 74, May-June 2012, pp. 1-19.

[18] Infeld, Samantha I., Optimization of mission design for constrained libration point space missions, ProQuest Dissertations And Theses; Thesis (Ph.D.), Stanford University, 2006, Publication Number: AAI3209042; ISBN: 9780542570926; Source: Dissertation Abstracts International, Volume: 67-02, Section: B, page: 1001.; 133 p.

[19] Tae Soo No, JiMarn Lee, Gyeong Eon Jeon, Daero Lee, and Ghangho Kim, "A Study on Earth-Moon Transfer Orbit Design", Int'l J. of Aeronautical \& Space Sci., Volume 13, No. 1, 2012, pp. 106-116. 
[20] Parker, Jeff., Dynamical Systems Theory Applied to Spacecraft Mission Design, Dec 6, 2012.

[21] Kyle Wolma, The Use of Lagrange Points for Lunar Exploration, Settlement, and Support, ASEN5050, Colorado University.

[22] STK Tutorial, Trajectory Design with STK/Astorgator : Earth-Moon L1 Trajectory Tutorial.

[23] Alfano, S., "A Numerical Implementation of Spherical Object Collision Probability", The Journal of Astronautical Sciences, Vol. 53, No. 1, January-March 2005, pp. 103-109.

[24] Michael R. Phillips, Spacecraft Collision Probability Estimation For Rendezvous and Proximity and Operation,
Utah State University, 2012.

[25] Alfano S, "Collision avoidance maneuver planning tool", in Proceedings of the 15th AAS/AIAA Astrodynamics Specialist Conference, Lake Tahoe, CA, 7-11 Aug 2005.

[26] Alfano S, "Review of conjunction probability methods for short-term encounters", in Proceedings of the AAS/AIAA Space Flight Mechanics Meeting, Sedona, AZ, 28 Jan-1 Feb 2007.

[27] Eun-Hyouek Kim, Hae-Dong Kim, Hak-Jung Kim, "Optimal Solution of Collision Avoidance Maneuver with Multiple Space Debrisc, Journal of Space OperationsLetters, Vol. 9, No. 3, July—September 2012. 